\title{
Kinetics of alkaline hydrolysis of synthetic organic
}

\section{esters}

Do Young Maeng ${ }^{1}$ and V. Faye McNeill ${ }^{1,2 *}$

(1) Department of Chemical Engineering, Columbia University, New York, NY USA 10027

(2) Department of Earth and Environmental Sciences, Columbia University, New York, NY

USA 10027

* to whom correspondence should be addressed.vfm2103@columbia.edu +1-212-854-2869

\section{TOC Art}

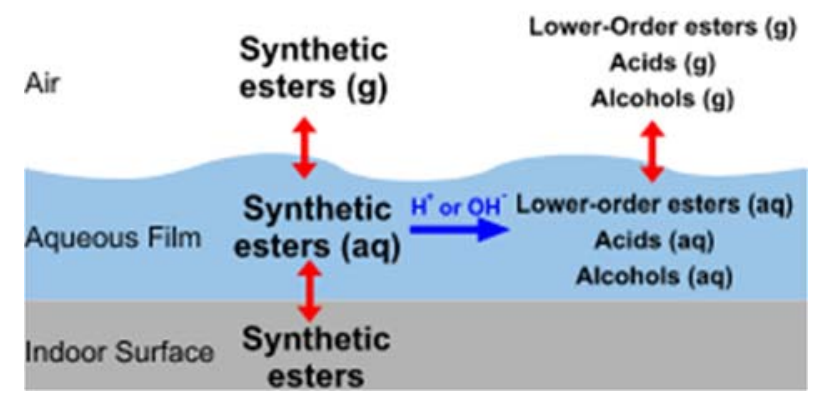

\begin{abstract}
The hydrolysis of phthalates and other synthetic organic esters (SEs) in the indoor environment may be connected with poor indoor air quality and "sick building syndrome." We have made laboratory measurements of the alkaline hydrolysis kinetics and mechanisms of 2,2,4-trimethyl-
\end{abstract}


1,3-pentanediol monoisobutyrate (TMPD-MIB), butylparaben (BP), bis(2-ethylhexyl) adipate (DEHA), and butyl benzyl phthalate (BBzP) in bulk aqueous solutions at room temperature, using UPLC-QToF-MS to detect the reactants over the course of the reactions. The determined alkaline hydrolysis second-order rate constants for TMPD-MIB, BP, DEHA, and BBzP were $(9.85 \pm 0.15)$ $\times 10^{-3} \mathrm{M}^{-1} \mathrm{~s}^{-1},(1.24 \pm 0.03) \times 10^{-4} \mathrm{M}^{-1} \mathrm{~s}^{-1},(4.86 \pm 0.11) \times 10^{-4} \mathrm{M}^{-1} \mathrm{~s}^{-1}$, and $(5.95 \pm 0.08) \times 10^{-2} \mathrm{M}^{-}$

${ }^{1} \mathrm{~s}^{-1}$, respectively. The results suggest that hydrolysis may be a major degradation pathway for these compounds in the indoor environment under alkaline conditions.

\section{Introduction}

Synthetic organic esters (SEs) are ubiquitous indoor pollutants used as additives in numerous consumer products and building materials. Phthalates, adipates, and isobutyrates are used as plasticizers which enhance the flexibility of the materials such as electronic cables, adhesives, PVC flooring, and latex paint. ${ }^{1,2}$ Parabens provide microbial resistance as preservatives, and are therefore commonly found in cosmetics and food packaging. ${ }^{3}$ Although SEs enter the indoor environment in the condensed phase (e.g. physically bound in plastic materials), they leach out and partition to the gas phase and/or dissolve in nanoscale aqueous films. ${ }^{4-6}$ Such aqueous films, formed in damp conditions, can be acidic from the uptake of gases, or basic when in contact with alkaline surfaces such as concrete. ${ }^{7}$ Esters are vulnerable to reaction with water molecules (hydrolysis) in the gas or aqueous phase, or on surfaces. ${ }^{6,8}$ Due to relatively slow gas-phase hydrolysis rates, and the fact that ester hydrolysis may be either acid or base-catalyzed, hydrolysis on surfaces and in aqueous films is likely the most important pathway for hydrolytic degradation of SEs indoors.

Direct exposure to certain phthalates and parabens is known to be harmful for human health, 
with effects including reproductive toxicity, endocrine disruption, and allergy sensitization., ${ }^{9,10}$ The degradation products of these species in the indoor environment also pose additional health concerns. Products of SE hydrolysis include alcohols, organic acids (or carboxylate salt, under alkaline conditions), or in the case of di- and tri- esters, smaller ester compounds. Elevated gasphase concentrations of volatile organic compounds (VOCs) from various indoor sources, including the alcohol degradation products of ester hydrolysis, are associated with sick building syndrome (SBS) - a condition where building occupants experience acute health effects and discomfort associated with their time indoors. ${ }^{11}$ For example, exposure to 2-ethyl-1-hexanol, an alcohol generated during the hydrolytic degradation of bis(2-ethylhexyl) phthalate (DEHP) and bis(2-ethylhexyl) adipate (DEHA), has been connected to SBS and asthma-like symptoms, especially in buildings with signs of dampness or high humidity. ${ }^{12,13}$

Despite the concerning implications for indoor air quality, the kinetics of SE hydrolysis remain largely unknown. Thus, the objective of this study was to determine the alkaline hydrolysis kinetics of four synthetic esters commonly found in the indoor environment: 2,2,4-trimethyl-1,3pentanediol monoisobutyrate (TMPD-MIB, also known as Texanol $\left.{ }^{\circledR}\right)$, butylparaben (BP), bis(2ethylhexyl) adipate (DEHA), and butyl benzyl phthalate (BBzP).

\section{Materials and Methods}

Chemicals. All chemicals were used as received. The reactant esters were 2,2,4-trimethyl-1,3pentanediol monoisobutyrate (TMPD-MIB, 99\% purity, Sigma Aldrich), butylparaben (BP, 99\% purity, Thermo Fisher Scientific), bis(2-ethylhexyl) adipate (DEHA, 99\% purity, Sigma Aldrich), and butyl benzyl phthalate (BBzP, 98\% purity, Sigma Aldrich). Water (HPLC grade, Sigma Aldrich) and sodium hydroxide ( $\mathrm{NaOH}$, extra pure, $50 \mathrm{wt} \%$ solution in water, Thermo Fisher Scientific) were used to prepare the solvent mixtures, with the addition of isopropyl 
alcohol (Optima LC/MS grade, Thermo Fisher Scientific) for DEHA and BBzP hydrolyses. Acetic acid (glacial, Optima, Thermo Fisher Scientific) was used to neutralize the aliquots after their extraction. The UPLC mobile phase solvents (listed in the Supporting Information) were of Optima LC/MS grade and purchased from Thermo Fisher Scientific.

Kinetic Procedure. Second-order alkaline hydrolysis rate constants were determined by observing the hydrolytic degradation of TMPD-MIB, BP, DEHA, and BBzP over time. The product distributions of the hydrolysis reactions are provided in the Supporting Information.

The employed kinetic method outlined below is based on previous investigations on the alkaline hydrolysis of phthalate esters. ${ }^{14}$ The experiments were performed in triplicate for each of the esters studied.

A bulk aqueous solution of $0.02 \sim 0.1 \mathrm{M}$ sodium hydroxide $(\mathrm{NaOH})$ was mixed with a dilute aqueous solution of each ester using a vortex mixer, and the reacting solution was contained in a sealed, sterile polypropylene tube at room temperature monitored with a temperature meter (Vaisala, HM40) throughout the reaction. The $\mathrm{pH}$ of the solution was measured using a $\mathrm{pH}$ meter (Thermo Scientific, Orion 4-Star pH/DO Benchtop Meter) to quantify the concentration of hydroxide ion $\left(\mathrm{OH}^{-}\right)$. For DEHA and BBzP, which have relatively low water solubility $\left(<10^{-5}\right.$ M), the solutions were prepared with $1 \%$ isopropyl alcohol in volume in order to facilitate dissolution. Aliquots were drawn from each reaction solution at predetermined time intervals and immediately quenched with diluted aqueous acetic acid for UPLC-MS analysis.

The alkaline hydrolysis of esters is known to follow second-order kinetics-first-order with respect to each of the reactants, the ester and $\mathrm{OH}^{-} \cdot{ }^{15}$ Because $\mathrm{OH}^{-}$was in large excess, the studied reactions were assumed to follow pseudo first-order kinetics. Therefore, the pseudo first-order 
rate constants were determined as the slopes of least squares regression lines of the natural logarithm of concentration ratio versus time. They were then divided by the $\mathrm{OH}$ - concentrations to calculate the second-order rate constants of the reactions.

UPLC-MS Analysis. TMPD-MIB, BP, DEHA, and BBzP were quantified using an ACQUITY Ultra-Performance Liquid Chromatography system (Waters Corporation, UPLC H-Class PLUS) in tandem with a quadrupole time-of-flight mass spectrometer (Waters Corporation, Xevo G2XS QToF MS). All esters were separated on an ACQUITY UPLC CSH C18 Column (1.7 $\mu \mathrm{m}$, $2.1 \times 50 \mathrm{~mm}$ ) (Waters Corporation). Signal interference from contamination was a noteworthy concern during the analysis because of the pervasiveness of the analyte esters in indoor environments. To mitigate this issue, an Isolator Column (Waters Corporation) was installed in the flow path prior to the injection port in UPLC to offset the background response from the sample analyte response. The QToF MS was operated with an electrospray ionization (ESI) source in positive mode for TMPD-MIB, DEHA, and BBzP analyses and in negative mode for BP analysis. Additional details regarding UPLC-QToF-MS operation and calibration are provided in the Supporting Information.

\section{Results and Discussion}

Plots of natural logarithm of normalized ester concentration versus time for all reactions are shown in Figure 1. Weighted linear least squares curves were fitted to each data set, from which the pseudo first-order rate constants were found as the slopes of the curves. Strong linearity across all plots indicated that the pseudo first-order assumption was valid for the studied kinetics. Second-order rate constants of the reactions, listed in Table 1, were determined as described in the previous section. 

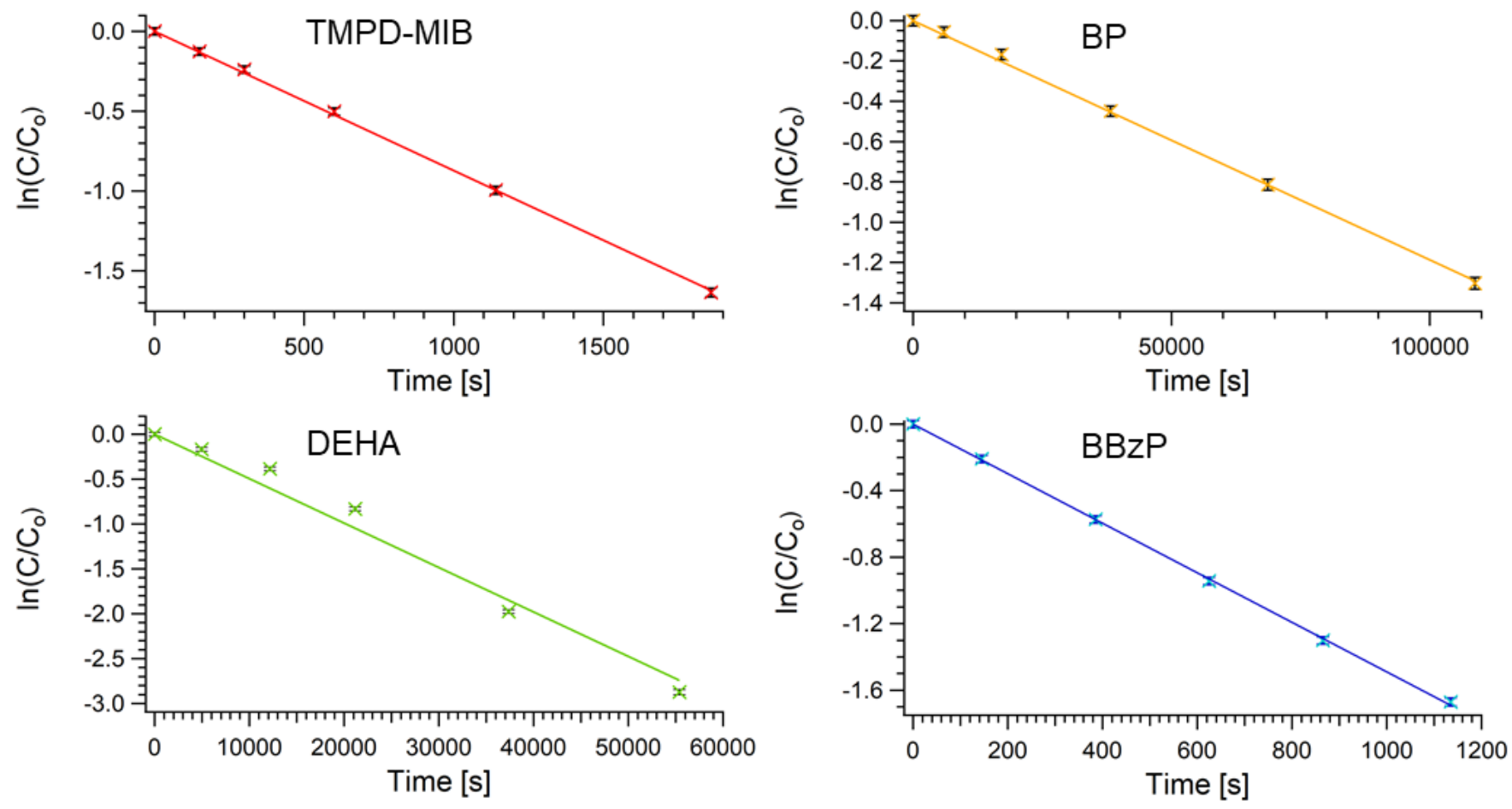

Figure 1. Hydrolytic degradation of TMPD-MIB (red), BP (yellow), DEHA (green), and BBzP (blue) over time in alkaline aqueous solution at room temperature. Error bars represent standard deviations based on triplicate measurements. 
Table 1. Alkaline Hydrolysis Second-Order Rate Constants of Synthetic Esters

\begin{tabular}{|c|c|c|c|c|}
\hline Ester & $\begin{array}{c}\mathrm{k} \\
{\left[\mathrm{M}^{-1} \mathrm{~s}^{-1}\right]}\end{array}$ & $\begin{array}{c}\text { Temperature } \\
{\left[{ }^{\circ} \mathrm{C}\right]}\end{array}$ & $\mathrm{pH}$ & Reference \\
\hline 2,2,4-Trimethyl-1,3-pentanediol monoisobutyrate (TMPD-MIB) & $(9.85 \pm 0.15) \times 10^{-3}$ & $20.13 \pm 0.06$ & $12.95 \pm 0.01$ & this study \\
\hline Butylparaben (BP) & $(1.24 \pm 0.03) \times 10^{-4}$ & $20.86 \pm 0.04$ & $12.99 \pm 0.01$ & this study \\
\hline Bis(2-ethylhexyl) adipate (DEHA) & $(4.86 \pm 0.11) \times 10^{-4}$ & $20.58 \pm 0.03$ & $13.01 \pm 0.01$ & this study \\
\hline Methylparaben & $(8.7 \pm 0.3) \times 10^{-4}$ & $30 \pm 0.1$ & $13 *$ & 16 \\
\hline Ethylparaben & $(4.2 \pm 0.2) \times 10^{-4}$ & $30 \pm 0.1$ & $13 *$ & 16 \\
\hline Propylparaben & $(3.0 \pm 0.1) \times 10^{-4}$ & $30 \pm 0.1$ & $13 *$ & 16 \\
\hline Diethyl phthalate & $(2.5 \pm 0.2) \times 10^{-2}$ & $30.00 \pm 0.05$ & 10 to $12^{\dagger}$ & 14 \\
\hline Di-n-butyl phthalate & $(1.0 \pm 0.05) \times 10^{-2}$ & $30.00 \pm 0.05$ & 10 to $12^{\dagger}$ & 14 \\
\hline
\end{tabular}

*: Estimated from the provided $\mathrm{NaOH}$ concentrations ${ }^{14,16}$ 
It should be noted that the tabulated rate constants are associated with the overall degradation rate of each species. For DEHA, which has two identical ester moieties, both reaction sites are equivalent in terms of their contribution to the rate of hydrolysis. For BBzP, however, its ester moieties have different chemical structures, resulting in two distinct pathways for its hydrolysis reaction. The benzyl ester is likely much more vulnerable to hydrolysis than the butyl ester for reasons described in detail below, but further study is needed to determine the kinetics associated with each ester moiety within the compound.

Neglecting minor differences in reaction temperature, the alkaline hydrolysis rate constant of BBzP was the fastest, followed by those of TMPD-MIB, DEHA, and lastly BP. The differences in hydrolysis reactivity are explained by comparing the substituents of the esters. The substituents can either hinder or facilitate the rate-determining nucleophilic attack of $\mathrm{OH}^{-}$on the carbonyl carbon of the ester by exerting polar and steric effects. The phenyl substituent of BBzP is more electron-withdrawing than the alkyl substituents of TMPD-MIB and DEHA, increasing the electrophilicity of the reaction site and consequently promoting the reaction. In contrast, the phenol substituent of BP is electron-donating instead and significantly slows the rate of hydrolysis. Alkyl chain substituents are found in both TMPD-MIB and DEHA, but those in TMPD-MIB are shorter and therefore exert a weaker steric effect than those in DEHA, resulting in TMPD-MIB being more reactive than DEHA.

Alkaline conditions are representative of aqueous films in contact with concrete, which have been implicated in SBS episodes involving SE hydrolysis. ${ }^{713}$ Although aqueous films indoors could also potentially be acidic, ${ }^{7}$ and ester hydrolysis could be acid-catalyzed, no data currently exist for those processes. Alkaline hydrolysis kinetics of other parabens and phthalates - ester families encompassing BP and BBzP, respectively—have been investigated in the past. ${ }^{14,16} \mathrm{BP}$ 
and $\mathrm{BBzP}$ alkaline hydrolysis kinetics results are compared to those of other members of their respective families in Table 1. Taking into account the aforementioned polar and steric effects of substituents, the kinetic results of $\mathrm{BP}$ and $\mathrm{BBzP}$ reasonably fit the trend among the literature values of their respective families. The reaction rate constant of BP is slower than those of other paraben species with smaller alkyl chains. On the contrary, the reaction rate constant of BBzP is significantly faster than other phthalates with alkyl chain substituents because of the electronwithdrawing phenyl group in BBzP.

SEs are typically short-lived in the aqueous phase under highly alkaline conditions ( $\mathrm{pH} 13)$ at room temperature $\left(20 \sim 21^{\circ} \mathrm{C}\right)$ as illustrated by their calculated hydrolysis half-lives in Table 2 . The half-life calculations are described in detail in the Supporting Information. In circumstances where the aqueous film is in direct contact with surfaces such as fresh concrete, such highly alkaline conditions are attainable as the $\mathrm{pH}$ typically varies from 11 to $13 .{ }^{13}$ The results suggest that, in damp indoor environments with insufficient ventilation and alkaline surfaces, the generation of volatile compounds from SE hydrolysis could contribute to poor indoor air quality.

Table 2. Half-Lives of Synthetic Esters at pH 13 and Room Temperature

\begin{tabular}{lc}
\hline \multicolumn{1}{c}{ Ester } & $\begin{array}{c}\text { Half-life } \\
{[\mathrm{hr}]}\end{array}$ \\
\hline 2,2,4-Trimethyl-1,3-pentanediol monoisobutyrate (TMPD-MIB) & 0.195 \\
Butylparaben (BP) & 15.5 \\
Bis(2-ethylhexyl) adipate (DEHA) & 3.96 \\
Butyl benzyl phthalate (BBzP) & 0.0324 \\
\hline
\end{tabular}


Besides $\mathrm{pH}$, the hydrolytic degradation of esters and the subsequent production of volatile organic compounds (VOCs) is dependent on other important physicochemical processes, such as ester migration from surfaces (leaching) and air-water partitioning of esters and their hydrolysis

products. More data on these processes, along with information on surface film properties, are needed before substantial claims can be made on the impact of SE hydrolysis on indoor air. Further study on these topics, as well as modeling which couples the gas and aqueous phase chemistry with the multiphase mass transfer, is necessary to understand the relationship between building-related illnesses and the hydrolysis of synthetic esters.

\section{Supporting Information}

Reaction scheme. Further details on the operation of the UPLC-QToF-MS and calibration. Halflife calculation.

\section{Acknowledgements}

We acknowledge the Alfred P. Sloan Foundation Chemistry of the Indoor Environment program for funding and Dr. Brandon Fowler for assistance with UPLC-QToF-MS method development.

\section{References}

(1) Godwin, A. D. Plasticizers. In Applied Polymer Science: 21st Century; Pergamon, 2000; pp 157-175. https://doi.org/10.1016/B978-008043417-9/50011-8. 
(2) Lin, C. C.; Corsi, R. L. Texanol® Ester Alcohol Emissions from Latex Paints: Temporal Variations and Multi-Component Recoveries. Atmos. Environ. 2007, 41 (15), 3225-3234. https://doi.org/10.1016/j.atmosenv.2006.07.057.

(3) Centers for Disease Control and Prevention. Parabens Factsheet https://www.cdc.gov/biomonitoring/Parabens_FactSheet.html.

(4) Weschler, C. J.; Nazaroff, W. W. Semivolatile Organic Compounds in Indoor Environments. Atmos. Environ. 2008, 42 (40), 9018-9040. https://doi.org/10.1016/j.atmosenv.2008.09.052.

(5) Till, D. E.; Reid, R. C.; Schwartz, P. S.; Sidman, K. R.; Valentine, J. R.; Whelan, R. H. Plasticizer Migration from Polyvinyl Chloride Film to Solvents and Foods. Food Chem. Toxicol. 1982, 20 (1), 95-104. https://doi.org/10.1016/S0278-6915(82)80016-1.

(6) Duncan, S. M.; Sexton, K. G.; Turpin, B. J. Oxygenated VOCs, Aqueous Chemistry, and Potential Impacts on Residential Indoor Air Composition. Indoor Air 2018, 28 (1), 198212. https://doi.org/10.1111/ina.12422.

(7) Ault, A. P.; Grassian, V. H.; Carslaw, N.; Collins, D. B.; Destaillats, H.; Donaldson, D. J.; Farmer, D. K.; Jimenez, J. L.; McNeill, V. F.; Morrison, G. C.; et al. Indoor Surface Chemistry: Developing a Molecular Picture of Reactions on Indoor Interfaces. Chem 2020, 1-16. https://doi.org/10.1016/j.chempr.2020.08.023.

(8) Takashima, K.; Riveros, J. M. Gas-Phase Pathways for Ester Hydrolysis. J. Am. Chem. Soc. 1978, 100 (19), 6128-6132. https://doi.org/10.1021/ja00487a027.

(9) Schettler, T.; Skakkebæk, N. E.; De Kretser, D.; Leffers, H. Human Exposure to 
Phthalates via Consumer Products. Int. J. Androl. 2006, 29 (1), 134-139.

https://doi.org/10.1111/j.1365-2605.2005.00567.x.

(10) Reinmuth-Selzle, K.; Kampf, C. J.; Lucas, K.; Lang-Yona, N.; Fröhlich-Nowoisky, J.;

Shiraiwa, M.; Lakey, P. S. J.; Lai, S.; Liu, F.; Kunert, A. T.; et al. Air Pollution and

Climate Change Effects on Allergies in the Anthropocene: Abundance, Interaction, and

Modification of Allergens and Adjuvants. Environ. Sci. Technol. 2017, 51 (8), 4119-4141.

https://doi.org/10.1021/acs.est.6b04908.

(11) Brinke, J. Ten; Selvin, S.; Hodgson, A. T.; Fisk, W. J.; Mendell, M. J.; Koshland, C. P.;

Daisey, J. M. Development of New Volatile Organic Compound (VOC) Exposure Metrics and Their Relationship to "Sick Building Syndrome" Symptoms. Indoor Air 1998, 8 (3), 140-152. https://doi.org/10.1111/j.1600-0668.1998.t01-1-00002.x.

(12) Norback, D.; Wieslander, G.; Nordstrom, K.; Walinder, R. Asthma Symptoms in Relation to Measured Building Dampness in Upper Concrete Floor Construction, and 2-Ethyl-1Hexanol in Indoor Air. Int. J. Tuberc. Lung Dis. 2000, 4 (11), 1016-1025.

(13) Björk, F.; Eriksson, C. A.; Karlsson, S.; Khabbaz, F. Degradation of Components in Flooring Systems in Humid and Alkaline Environments. Constr. Build. Mater. 2003, 17 (3), 213-221. https://doi.org/10.1016/S0950-0618(02)00036-3.

(14) Wolfe, N. L.; Steen, W. C.; Burns, L. A. Phthalate Ester Hydrolysis: Linear Free Energy Relationships. Chemosphere 1980, 9 (7-8), 403-408. https://doi.org/10.1016/00456535(80)90023-5.

(15) Kirby, A. J. Hydrolysis and Formation of Esters of Organic Acids. In Comprehensive Chemical Kinetics; Bamford, C. H., Tipper, C. F. H., Eds.; Elsevier, 1972; Vol. 10, pp 57- 
207. https://doi.org/10.1016/S0069-8040(08)70344-3.

(16) Shija, R.; Bruce Sunderland, V.; McDonald, C. Alkaline Hydrolysis of Methyl, Ethyl and n-Propyl 4-Hydroxybenzoate Esters in the Liquid and Frozen States. Int. J. Pharm. 1992, 80 (1-3), 203-211. https://doi.org/10.1016/0378-5173(92)90278-A. 